\title{
Os Temas Controversos na Educação Ambiental
}

\author{
Pedro Rocha dos Reis \\ Escola Superior de Educação do Instituto Politécnico de Santarém \\ Centro de Investigação em Educação da Faculdade de Ciências da \\ Universidade de Lisboa
}

Resumo: Vários autores acreditam que a discussão de questões controversas na sala de aula se revela extremamente útil quer na aprendizagem dos conteúdos, dos processos e da natureza da ciência e da tecnologia, quer no desenvolvimento cognitivo, social, político, moral e ético dos alunos (CACHAPUZ, PRAIA, PAIXÃO e MARTINS, 2000; MILLAR, 1997; NELKIN, 1992; REIS e PEREIRA, 1998; ZEIDLER e LEWIS, 2003). Estas opiniões têm sido apoiadas por diversas investigações (DORI, TAL e TSAUSHU, 2003; REIS, 1997, 1999, 2001; SADLER e ZEIDLER, 2004; SOLOMON, 1992; ZOHAR e NAMET, 2002). Apesar de todas as evidências empíricas das potencialidades educativas da discussão de questões controversas, estas actividades não fazem parte das experiências educativas da maioria das aulas de ciências, mesmo quando as questões controversas integram os conteúdos curriculares. Diversos factores inerentes à própria discussão, aos professores, aos alunos e a antipatias do sistema educativo desencorajam a utilização desta metodologia na sala de aula (LEVINSON e TURNER, 2001; MCGINNIS e SIMMONS, 1999; MITCHENER e ANDERSON, 1989; NEWTON, 1999; REIS, 2001, 2004; REIS e GALVÃO, 2004, 2005; SIMMONS e ZEIDLER, 2003; STRADLING, 1984). No entanto, conforme se discute neste artigo, as fortes convicções pessoais dos professores relativamente à importância da discussão de controvérsias em contexto de sala de aula, associadas à robustez do conhecimento de conteúdo disciplinar e do conhecimento didáctico sobre os alunos, as finalidades do ensino das ciências e as estratégias mais adequadas à concretização deste tipo de actividade, facilitam a ultrapassagem dos obstáculos referidos. 
Palavras-chave: Controvérsia. Educação Ambiental. Educação em Ciência.

Abstract: Several authors believe that the discussion of controversial issues in the classroom is particularly useful for learning the contents, the processes and the nature of science and the technology, and for pupils' cognitive, social, political, ethical and moral development (CACHAPUZ, PRAIA, PAIXÃO and MARTINS, 2000; MILLAR, 1997; NELKIN, 1992; REIS and PEREIRA, 1998; ZEIDLER and LEWIS, 2003). These opinions have been supported by research (DORI, TAL and TSAUSHU, 2003; REIS, 1997, 1999, 2001; SADLER and ZEIDLER, 2004; SOLOMON, 1992; ZOHAR and NAMET, 2002). Despite all the favourable opinions and empirical evidence concerning the educational potential of the discussion of controversial issues, the majority of science classrooms don't incorporate this type of activity (even when controversial issues comprise the curricular content). Several factors - inherent to the discussion itself, the teachers, the pupils and the educational system - discourage the use of this methodology in the classroom (LEVINSON and TURNER, 2001; MCGINNIS and SIMMONS, 1999; MITCHENER and ANDERSON, 1989; NEWTON, 1999; REIS, 2001, 2004; REIS and GALVÃO, 2004, 2005; SIMMONS and ZEIDLER, 2003; STRADLING, 1984). Nevertheless, as it is discussed in this article, professors' strong personal convinctions regarding the importance of the discussion of controversial issues in the classroom context, in association with the strenght of disciplinary content knowledge and pedagogical knowledge about students, as well as with the science teaching aims and the strategies more adjusted to the effectiveness of such activity, make it easier to surpass the referred barriers.

Keywords: Controversy. Environmental Education. Science Education.

Um dos argumentos mais frequentes a favor da literacia científica da população consiste na sua preparação para responder a questões sociais com uma dimensão científica ou tecnológica considerável (KOLSTOE, 2001; MILLAR, 1997). Considera-se que, numa sociedade democrática, a avaliação pública da ciência depende de indivíduos capazes de reconhecer o que está em causa numa controvérsia, de alcançar uma opinião informada e de participar em discussões, debates e processos de tomada de decisão. No entanto, existem evidências de que a educação científica não tem cumprido este objectivo. Os resultados de 
algumas investigações, centradas na forma como os adultos entendem e utilizam a ciência e na articulação da ciência com a acção prática, não são conciliáveis com a imagem de ciência transmitida pela escola formal (COLLINS e SHAPIN, 1986; JENKINS, 1997). Habitualmente, a escola formal retrata a ciência como coerente, objectiva, não problemática e claramente distinguível de actividades não científicas, veiculando um modelo de racionalidade científica que leva os alunos a pensarem que os métodos de investigação rigorosos revelam, de forma repetida, única e sem ambiguidades, factos verdadeiros sobre o mundo natural. No entanto, a realidade é bem diferente. Os especialistas discordam frequentemente dos pareceres uns dos outros, razão pela qual se torna extremamente importante a capacidade de avaliar a qualidade das informações apresentadas pelas facções envolvidas. Por vezes, em algumas controvérsias (por exemplo, em torno dos impactos ambientais e sociais da construção de usinas hidroeléctricas ou da co-incineração de resíduos tóxicos em usinas de cimento), as questões técnicas não obtêm resposta, apesar da vasta quantidade de informação técnica disponível, e as facções acusam-se de enviesamento na selecção dos dados que fundamentam as respectivas opiniões. Verifica-se, ainda, que controvérsias deste tipo não podem ser resolvidas simplesmente numa base técnica, pois envolvem outros aspectos, tais como hierarquizações de valores, conveniências pessoais, questões financeiras, entre outras.

Constata-se, assim, que a preparação dos alunos para a participação em processos avaliativos e decisórios sobre controvérsias socioambientais ou sociocientíficas não é uma tarefa simples. A avaliação das consequências e a correcção dos eventuais problemas resultantes de propostas científicas e tecnológicas requerem: a) um enquadramento de conhecimentos científicos indispensáveis à apropriação de conhecimentos mais pormenorizados sobre as questões em causa; b) conhecimentos metacientíficos sobre a natureza, as potencialidades e os limites da ciência; c) capacidades de pensamento crítico, tomada de decisões e resolução de problemas; d) atitudes e valores úteis à avaliação das dimensões ética e moral da ciência e da tecnologia; e e) vontade e confiança para lidarem com assuntos científicos. 
Vários autores defendem que o desenvolvimento deste conjunto de conhecimentos, capacidades e atitudes, necessário à compreensão das controvérsias suscitadas pelo impacto social e ambiental de propostas científicas e tecnológicas, deve ser efectuado através do envolvimento dos alunos na análise e discussão desse tipo de controvérsias (CACHAPUZ, PRAIA e JORGE, 2002; DRIVER, LEACH, MILLAR e SCOTT, 1996; MILLAR, 1997; REIS, 1997; REIS, 2004; ZEIDLER e LEWIS, 2003). Na sua opinião, a discussão destas questões controversas na sala de aula justifica-se não só pelos conhecimentos que promove acerca dos conteúdos, dos processos e da natureza da ciência e da tecnologia, mas também pelas potencialidades educativas deste tipo de interacção no desenvolvimento cognitivo, social, político, moral e ético dos alunos.

Existem controvérsias em todas as áreas do pensamento humano - na ciência, na história, na arte, na economia, na política, na teologia. No entanto, a forma como cada uma destas áreas de pensamento é representada no currículo académico nem sempre traduz os seus conteúdos ou a sua natureza controversa. Muitos professores reconhecem a ciência como um campo de controvérsias (tanto disputas académicas suscitadas, por exemplo, pela apresentação de diferentes propostas explicativas do mesmo fenómeno, como conflitos resultantes das implicações ambientais e sociais de propostas científicas ou tecnológicas), que evolui e se desenvolve através de conjectura e especulação, alimentadas pela própria controvérsia (BRIDGES, 1986). Contudo, a ciência escolar: a) é frequentemente apresentada como não problemática, livre de valores e não controversa, proporcionando uma imagem completamente distorcida do empreendimento científico e das suas relações com a tecnologia, a sociedade e o ambiente; e b) recorre pouco à controvérsia como forma de promover o desenvolvimento de capacidades e atitudes consideradas importantes para a cidadania (CACHAPUZ, PRAIA, PAIXÃO e MARTINS, 2000; DEARDEN, 1981; WELLINGTON, 1986).

Segundo Rudduck (1986), "uma questão é definida como controversa se as pessoas se encontram divididas sobre ela e se envolve juízos de valor que impossibilitam a sua resolução apenas através da análise das evidências ou da experiência" (p. 8). Um 
assunto controverso não pode ser resolvido apenas recorrendo a factos, dados empíricos ou vivências na medida em que envolve tanto factos como questões de valor. Gardner (1983) acrescenta que um assunto só poderá ser classificado de controverso se também for considerado importante por um número considerável de pessoas.

As controvérsias socioambientais ou as controvérsias sociocientíficas são consideravelmente diferentes do tipo de problemas geralmente realizado nas aulas de ciências. Frequentemente, estes últimos têm um âmbito bem delimitado e são algorítmicos, objectivos e accionados por conhecimento disciplinar disponível. O recurso a procedimentos ditos correctos traduz-se numa única resposta de tipo certo ou errado. Pelo contrário, as controvérsias socioambientais e sociocientíficas são pouco delimitadas, multidisciplinares, heurísticas, carregadas de valores (invocando, por exemplo, valores estéticos, ecológicos, morais, educacionais, culturais e religiosos) e marcadas pela ausência de conhecimento disciplinar disponível. Geralmente, o envolvimento neste tipo de problemas controversos conduz a diversas "soluções" alternativas, cada uma das quais com aspectos positivos e negativos. A partir destas diferentes propostas, toma-se uma decisão informada que, dada a impossibilidade de recurso a qualquer algoritmo para a avaliação das potencialidades e limitações, envolve a consideração e o desafio de opiniões.

São várias as razões que levam diversos autores a defenderem a inclusão de actividades de discussão de assuntos controversos nos currículos. Stradling, Noctor e Bains (1984) acreditam que estas actividades se justificam tanto pelos conhecimentos científicos como pelas capacidades que promovem. A pesquisa de informação, a detecção de incoerências, a avaliação da idoneidade das fontes, a comunicação de informação recolhida e/ou de pontos de vista, a fundamentação de opiniões, o poder de argumentação e o trabalho cooperativo constituem exemplos de capacidades que podem ser desenvolvidas através da discussão de controvérsias.

Outros autores destacam a importância da discussão de controvérsias socioambientais ou de controvérsias sociocientíficas na construção de uma imagem mais real e humana do 
empreendimento científico (e das suas múltiplas interacções com a tecnologia, a sociedade e o ambiente) e na promoção da literacia científica indispensável a uma cidadania responsável (MILLAR e OSBORNE, 1998; WANG e SCHMIDT, 2001). DUSCHL (2000) está convicto de que a participação dos cidadãos em processos decisórios relacionados com questões científicas e tecnológicas depende da compreensão das dinâmicas sociais, cognitivas e epistémicas da ciência. Portanto, defende acerrimamente um ensino das ciências promotor de reflexão sobre a natureza da ciência e das inter-relações entre ciência, tecnologia, sociedade e ambiente.

Para Dearden (1981), uma abordagem completa de qualquer disciplina passa pela referência aos seus elementos controversos. A título de exemplo, aponta a não inclusão destes elementos nos currículos da área científica como responsável pela transmissão de ideias distorcidas sobre a ciência, frequentemente descrita como não controversa, neutra, desinteressada, altruísta. Nelkin (1992) considera que, no decurso de uma disputa, os interesses, as preocupações e as motivações dos vários agentes são claramente revelados. Logo, defende que a análise dos detalhes de uma controvérsia proporciona aos alunos: a) conhecimentos sobre o tipo de raciocínio que motiva os governos, os cientistas e os grupos de protesto; e b) uma compreensão realista de uma política científica e tecnológica, do seu contexto social e político e do seu impacto no público em geral ou em determinadas comunidades. Considera, também, que as disputas realçam: a) as contradições inerentes a muitas decisões sobre ciência e tecnologia; b) os problemas do desenvolvimento de linhas de acção, na ausência de consensos definitivos sobre os eventuais riscos (nomeadamente ambientais); e c) as questões éticas de opções que envolvem conflitos de valores.

Rudduck (1986) acredita que a exploração activa desta metodologia pode ajudar a desenvolver o pensamento crítico e a independência intelectual. Para tal, defende que os alunos devem ser ajudados a encarar a controvérsia convictos do seu direito de formular opiniões e de tomar decisões, e não na expectativa de que qualquer autoridade possa decidir e resolver em seu lugar.

Segundo Berman (1997), a escola é, na sua essência, uma microssociedade (com uma estrutura política explícita e implícita) 
que proporciona aos alunos um conjunto de experiências sociais, políticas e morais. Tanto a escola como a sala de aula são comunidades (com uma estrutura governativa, um sistema de justiça, convenções sociais, conflitos e padrões de vida e de trabalho) constituídas por pessoas de diferentes idades, culturas e capacidades. Logo, considera que a responsabilidade social pode ser desenvolvida neste contexto experiencial, não apenas pelo que é ensinado, mas também pela forma como é ensinado e como a sala de aula e a escola são estruturadas. $\mathrm{Na}$ sua opinião, existem dois factores indispensáveis ao desenvolvimento da responsabilidade social em contexto escolar: a) um clima de sala de aula que convide a expressão aberta das opiniões dos alunos; e b) o recurso à controvérsia e ao conflito. Berman (1997) acredita que o conflito e a controvérsia desempenham um papel importante no desenvolvimento da consciência e da eficácia política: "os jovens necessitam de compreender o conflito e o processo da sua resolução no âmbito do nosso sistema político, bem como experimentar o envolvimento directo em conflito político" (p. 395). A inexperiência relativamente ao conflito e à controvérsia leva os alunos a evitá-los, dificultando-lhes a assunção de papéis políticos.

Vários autores destacam a importância da discussão de controvérsias tanto na formulação como na avaliação-reformulação de opiniões e crenças e, portanto, na educação moral e cívica (BERKOWITZ e SIMMONS, 2003; DEDECKER, 1986, 1987; HARRISON, 1993; LICKONA, 1991; REIS, 1997; RUDDUCK, 1986; ZEIDLER, 2003; SADLER e ZEIDLER, 2004). Acreditam que este tipo de experiência educativa ajuda os alunos a compreenderem as situações sociais, os actos humanos e as questões de valores por eles suscitadas. O envolvimento dos alunos na análise e discussão de problemas morais no domínio das interacções ciência-tecnologia-sociedade-ambiente, cuidadosamente seleccionados, permite desenvolver, simultaneamente, capacidades de raciocínio lógico e moral e uma compreensão mais profunda de aspectos importantes da natureza da ciência. As controvérsias revelam-se úteis na transformação das aulas de ciências num "microcosmos social" promotor do desenvolvimento holístico dos alunos nos domínios cognitivo, social, moral, ético e emocional (ZEIDLER e KEEFER, 2003). 
As potencialidades educativas da discussão de controvérsias têm sido evidenciadas em diversas investigações. Uma série de estudos sobre o impacto educativo do conflito e da controvérsia na sala de aula permitiu constatar que a sua utilização, no âmbito de uma estrutura de aprendizagem cooperativa, promove a motivação, a pesquisa e o intercâmbio de informação, a reavaliação das posições individuais, atitudes positivas acerca da controvérsia, sentimentos de auto-estima, relações de apoio entre os alunos, bem como a apreciação dos conteúdos e das experiências de ensino JOHNSON e JOHNSON, 1995; JOHNSON, BROOKER, STUTZMAN, HULTMAN e JOHNSON, 1985; LOWRY e JOHNSON, 1981; REIS, 1997; SMITH, JOHNSON e JOHNSON, 1984; TJOSVOLD, JOHNSON e LERNER, 1981).

Outras investigações (referidas em BERKOWITZ e SIMMONS, 2003) evidenciaram a importância da discussão de questões morais na reanálise das concepções pessoais e dos processos de pensamento e, consequentemente, na estimulação do desenvolvimento das estruturas de raciocínio sociomoral dos alunos. Mais uma vez, a chave deste desenvolvimento reside no confronto interpessoal e intrapessoal de ideias diferentes. Solomon (1992), num estudo efectuado com alunos de 17 anos que frequentavam cursos de Ciência, Tecnologia e Sociedade em 14 escolas inglesas (e que envolveu a gravação áudio e a análise da interacção verbal estabelecida durante a discussão de programas de televisão sobre temas controversos), verificou que a utilização repetida de actividades de discussão em pequeno grupo contribui positivamente para os processos de argumentação e de reflexão, constituindo uma experiência agradável e significativa em termos de aprendizagem. Na sua opinião, a participação real e genuína dos alunos nas discussões (através da partilha de experiências pessoais, bem como de dúvidas e incertezas sobre novos conhecimentos), apoiada num ambiente de confiança, torna este tipo de actividade de tal forma significativo e memorável que, mesmo passadas duas ou mais semanas, os alunos são capazes de relatar opiniões complexas discutidas em grupo.

Algumas investigações permitiram constatar um impacto bastante significativo da discussão de estudos de caso sobre controvérsias, relacionadas com implicações ambientais e morais de 
propostas na área da biotecnologia, no nível de conhecimentos científicos e técnicos sobre as temáticas em causa e de capacidades de pensamento de nível elevado (nomeadamente, formulação de questões e argumentação) (DORI, TAL e TSAUSHU, 2003; YERRICK, 2000; ZOHAR e NAMET, 2002). Nestes estudos, também foi evidente o interesse suscitado, entre professores e alunos, tanto pela metodologia (discussão de estudos de caso) como pelo tema das actividades utilizadas. Outros estudos revelaram potencialidades da análise e discussão de controvérsias sociocientíficas: a) na estimulação da interacção e no aprofundamento e melhoramento das relações interpessoais dos intervenientes (REIS, 1997, 1999; REIS e PEREIRA, 1998); b) no desenvolvimento de capacidades de raciocínio lógico e moral e de uma compreensão mais profunda da natureza da ciência (REIS, 1997, 1999; REIS e PEREIRA, 1998; SADLER e ZEIDLER, 2004; ZEIDLER, WALKER, ACKETT e SIMMONS, 2003); e c) na aprendizagem de conteúdos de ciência e de tecnologia, em estreita relação com o próprio contexto social que os torna significativos (MARTÍN GORDILLO e OSORIO, 2003). A disponibilização de informação relevante e diversificada sobre o assunto em discussão (programas de televisão, entrevistas, colóquios, trechos de livros, jornais e revistas) revela-se decisiva na estimulação da interacção e na exploração e aprofundamento de diferentes perspectivas. Sem ela, os alunos limitam-se a discutir as suas perspectivas, frequentemente limitadas (REIS, 1997).

Apesar de todas as evidências empíricas das potencialidades educativas da discussão de questões controversas, estas actividades não fazem parte das experiências educativas da maioria das aulas de ciências, mesmo quando as questões controversas integram os conteúdos curriculares. Verifica-se, também, que as opiniões bastante favoráveis de alguns professores relativamente à realização de actividades de discussão de questões sociocientíficas nas aulas de ciências acabam por ter um impacto modesto nas práticas de sala de aula (REIS, 2004).

Os professores reconhecem o potencial educativo destas actividades, nomeadamente: a) no desenvolvimento de capacidades e na construção de conhecimentos relevantes para a vida e indispensáveis à compreensão e à avaliação pública das 
controvérsias socioambientais ou das controvérsias sociocientíficas; b) na estimulação da curiosidade e do interesse dos alunos nas tarefas escolares; c) no estabelecimento de conexões entre a ciência abordada na escola e a ciência divulgada pelos meios de comunicação social; d) na veiculação de uma noção de ciência como empreendimento humano, influenciado por valores e interesses, que envolve investigação, análise, partilha e discussão de ideias e opiniões frequentemente controversas (REIS, 2001; 2004). Contudo, constata-se que este tipo de actividade está longe de ser uma prática comum nas suas aulas. Diversos factores (inerentes ao próprio processo de discussão, aos professores, aos alunos e ao sistema educativo) dificultam a sua concretização:

1. O receio dos eventuais protestos dos encarregados de educação e de uma possível falta de controlo durante as discussões (LICKONA, 1991; STRADLING, 1984);

2. A grande quantidade de termos, conceitos, factos e teorias incluídos nos currículos de ciências, que dificulta a criação dos tempos indispensáveis à concretização deste tipo de actividades (LEVINSON e TURNER, 2001; REIS, 2001, 2004);

3. A inexistência de referências explícitas ou de sugestões de abordagem relativamente à discussão de assuntos controversos em alguns currículos de ciências, associada à dificuldade evidenciada por alguns professores na identificação de tópicos que se adéquem ou permitam a realização de actividades de discussão (LEVINSON e TURNER, 2001; REIS, 2001, 2004);

4. A dificuldade de alguns professores em abdicarem de comportamentos autoritários, inconciliáveis com a postura liberal necessária a uma actividade de discussão (REIS, 2004);

5. A falta de materiais de ensino com sugestões de actividades adequadas às diferentes disciplinas de ciências (LEVINSON e TURNER, 2001; REIS, 2001, 2004);

6. O tipo de exame nacional proposto, com grande ênfase na memorização e fraca incidência em aspectos de 
natureza processual ou epistemológica da ciência, induzindo práticas de sala de aula pouco centradas na análise crítica e na discussão (nomeadamente, da natureza e da dinâmica do empreendimento científico) (LEVINSON e TURNER, 2001; MCGINNIS e SIMMONS, 1999; NEWTON, 1999; REIS, 2001, 2004);

7. O facto de as actividades de discussão de controvérsias socioambientais e de controvérsias sociocientíficas serem, por vezes, encaradas como meras "conversas informais extra-programa" (REIS, 2001, 2004);

8. A reduzida experiência dos alunos na discussão em sala de aula e a consequente falta de competências para a realização desse tipo de actividade (REIS, 2004);

9. A falta de conhecimento didáctico evidenciado por alguns professores relativamente à concepção, gestão e avaliação de actividades de discussão, em geral, e de temas controversos, em particular (LEVINSON e TURNER, 2001; MITCHENER e ANDERSON, 1989; REIS, 2004; STRADLING, 1984); e

10. A insuficiência de conhecimentos específicos por parte de alguns professores sobre: a) as complexidades técnicas inerentes às controvérsias; e b) os aspectos processuais e epistemológicos da ciência (LEVINSON e TURNER, 2001; NEWTON, 1999; PEDRETTI, 2003; REIS, 2004; SIMMONS e ZEIDLER, 2003).

No entanto, o nível de constrangimento imposto por estes factores varia entre os professores. Em determinados casos, as fortes convicções pessoais (relativamente à importância de uma abordagem explícita da controvérsia), associadas à robustez do conhecimento de conteúdo disciplinar e do conhecimento didáctico sobre os alunos, as finalidades do ensino das ciências e as estratégias mais adequadas à sua concretização, permitem ultrapassar estes obstáculos (REIS, 2001; 2004). As suas convicções e o seu conhecimento profissional conferem-lhes uma capacidade notável de interpretar o currículo de forma a abordarem os temas e a realizarem as actividades que consideram importantes $e$ 
relevantes. A capacidade de flexibilizar o currículo de acordo com os objectivos de aprendizagem desejados e as particularidades dos contextos de trabalho assume-se como um factor decisivo na realização de actividades de discussão de questões controversas.

Não basta que os professores possuam uma visão filosófica e sociologicamente mais rica e reflexiva sobre a ciência e as múltiplas interacções com a tecnologia, a sociedade e o ambiente, pois a coerência entre estas concepções e a prática de sala de aula é mediada por um conjunto diversificado e complexo de factores. Unicamente através da sua compreensão e da definição de estratégias para ultrapassar esses factores, será possível acalentar a esperança de uma educação científica mais real, humana e social e ambientalmente relevante.

\section{Referências bibliográficas}

BERKOWITZ, M. e SIMMONS, P. Integrating science education and character education: The role of peer discussion. In: ZEIDLER, D.L. (Ed.). The role of moral reasoning on socioscientific issues and discourse in science education. Dordrecht: Kluwer Academic Press, 2003, p. 117-138.

BERMAN, S. Children's social consciousness and the development of social responsibility. Albany: State University of New York Press, 1997.

BRIDGES, D. Dealing with controversy in the curriculum: A philosophical perspective. In: WELLINGTON, J. (Ed.). Controversial issues in the curriculum. Oxford: Basil Blackwell, 1986, p. 19-38.

CACHAPUZ, A.; PRAIA, J. e JORGE, M. Reflexão em torno do ensino das ciências: Contributos para uma nova orientação curricular - Ensino por pesquisa. Revista de Educação, IX(1), 2002, p. 69-79.

; PRAIA, J.; PAIXÃO, F. e MARTINS, I. Uma visão sobre o ensino das ciências no pós-mudança conceptual: Contributos para a formação de professores. Inovação, 13(2-3), p. 117-137, 2000.

COLLINS, H. M. e SHAPIN, S. Uncovering the nature of science. In: BROWN, J.; COOPER, A.; HORTON, T; TOATES, F. e ZELDIN, D. (Eds.). Science in Schools. London: Open University Press, 1986 [Reimpressão de Times Higher Education Supplement, 27 de Julho de 1984, 13]. 
DEARDEN, R. Controversial issues and the curriculum. Journal of Curriculum Studies, 13(1), p. 37-44, 1981.

DORI, Y, TAL, R. e TSAUSHU, M. Teaching biotechnology through case studies--Can we improve higher order thinking skills of nonscience majors? Science Education, 87(6), p. 767-793, 2003.

DRIVER, R., LEACH, J., MILLAR, R. e SCOTT, P. Young people's images of science. Buckingham: Open University Press, 1996.

DUSCHL, R. Making the nature of science explicit. In: MILLAR, R.; LEACH, J. \& OSBORN, J. (Eds.). Improving science education: The contribution of research. Buckingham: Open University Press, 2000, p. 187-206.

GARDNER, P. Another look at controversial issues and the curriculum. Journal of Curriculum Studies, 16, p. 179-185, 1983.

HARRISON, J. Citizenship and core and foundation subjects: Science. In: EDWARDS, J. e FOGELMAN, K. (Eds.). Developing citizenship in the curriculum. London: David Fulton, 1993, p. 47-50.

JENKINS, E. Towards a functional public understanding of science. In: LEVINSON, R.e THOMAS, J. Science today: Problem or crisis? London: Routledge, 1997, p. 137-150.

JOHNSON, R., BROOKER, C., STUTZMAN, J., HULTMAN, D. e JOHNSON, D. The effects of controversy, concurrence seeking, and individualistic learning on achievement and attitude change. Journal of Research in Science Teaching, 22, p. 197-205, 1985.

JOHNSON D. e JOHNSON, R. Creative controversy: Intellectual challenge in the classroom. Edina: Interaction Book Company, 1995.

KOLSTOE, S. Scientific literacy for citizenship: Tools for dealing with the science dimension of controversial socioscientific issues. Science Education, 85(3), p. 291-310, 2001.

LEVINSON, R. e TURNER, S. The teaching of social and ethical issues in the school curriculum, arising from developments in biomedical research: a research study of teachers. London: Institute of Education, University of London, 2001.

LICKONA, T. Educating for character: How our schools can teach respect and responsibility. New York: Bantam Books, 1991.

LOWRY, N. e JOHNSON, D. Effects of controversy on epistemic curiosity, achievement, and attitudes. Journal of Social Psychology, 115, p. 31-43, 1981. 
MARTÍN GORDILLO, M. e OSORIO, C. Educar para participar en ciencia y tecnología. Un proyecto para la difusión de la cultura científica. Revista Iberoamericana de Educación, 32, p. 165-210, 2003.

McGINNIS, J. e SIMMONS, P. Teachers' perspectives of teaching sciencetechnology-society in local cultures: A socio-cultural analysis. Science Education, 83, p. 179-211, 1999.

MILLAR, R. Science education for democracy: What can the school curriculum achieve? In: LEVINSON, R. e THOMAS, J. (Eds.). Science today: Problem or crisis? London: Routledge, p. 87-101, 1997.

e OSBORNE, J. Beyond 2000: Science education for the future. London: Kings College, 1998.

MITCHENER, C. e ANDERSON, R. Teachers' perspective: Developing and implementing an STS curriculum. Journal of Research in Science Teaching, 26(4), p. 351-369, 1989.

NELKIN, D. (Ed.). Controversy: politics of technical decisions. London: Sage Publications, 1992.

NEWTON, P. The place of argumentation in the pedagogy of school science. International Journal of Science Education, 21(5), p. 553-576, 1999.

PEDRETTI, E. Teaching science, technology, society and environment (STSE) education: Preservice teachers' philosophical and pedagogical landscapes. In: ZEIDLER, D.L. (Ed.). The role of moral reasoning on socioscientific issues and discourse in science education. Dordrecht: Kluwer Academic Press, 2003, p. 219239.

REIS, P. A promoção do pensamento através da discussão dos novos avanços na área da biotecnologia e da genética. Departamento de Educação da Faculdade de Ciências da Universidade de Lisboa, 1997 [Tese de mestrado, documento policopiado].

. O projecto "GENET": Biotecnologia, controvérsias e Internet. In: COELHO, A. C., ALMEIDA, A. F., CARMO, J. M. e SOUSA, M. N. (Eds.), Actas do VII Encontro Nacional de Educação em Ciências. Faro: Universidade do Algarve, Escola Superior de Educação, 1999, p. 454-458.

. O ensino das ciências através da discussão de controvérsias: realidade ou ficção? In: SILVA, B.D. e ALMEIDA, L.S. (Eds.). Actas do VI Congresso Galaico-Português de Psicopedagogia. Braga: Centro de Estudos em Educação e Psicologia da Universidade do Minho, 2001, p. 367-379. 
- Controvérsias sócio-científicas: Discutir ou não discutir? Percursos de aprendizagem na disciplina de Ciências da Terra e da Vida. Tese de Doutorado. Lisboa: Departamento de Educação da Faculdade de Ciências da Universidade de Lisboa, 2004.

e GALVÃO, C. The impact of socio-scientific controversies in portuguese natural science teachers' conceptions and practices. Research in Science Education, 34(2), p. 153-171, 2004.

e GALVÃO, C. Controvérsias sócio-científicas e prática pedagógica de jovens professores. Investigações em Ensino de Ciências, 10(2), 2005. Publicação da Universidade Federal do Rio Grande do Sul.

e PEREIRA, M. Discutindo o "admirável mundo novo". Inovação, 3, p. 45-59, 1998.

RUDDUCK, J. A strategy for handling controversial issues in the secondary school. In: J. J. Wellington (Ed.). Controversial issues in the curriculum. Oxford: Basil Blackwell, 1986, p. 6-18.

SADLER, T. e ZEIDLER, D. The morality of socioscientific issues: construal and resolution of genetic engineering dilemmas. Science Education, 88(1), p. 427, 2004.

SIMMONS, M. e ZEIDLER, D. Beliefs in the nature of science and responses to socioscientific issues. In: ZEIDLER, D.L. (Ed.). The role of moral reasoning on socioscientific issues and discourse in science education. Dordrecht: Kluwer Academic Press, 2003, p. 81-94.

SMITH, K., JOHNSON, D. e JOHNSON, R. Effects of controversy on learning in cooperative groups. Journal of Social Psychology, 122, p. 199-209, 1984.

SOLOMON, J. The classroom discussion of science-based social issues presented on television: knowledge, attitudes and values. International Journal of Science Education, 14, p. 431-444, 1992.

STRADLING, R. The teaching of controversial issues: an evaluation. Educational Review, 36(2), p. 121-129, 1984.

; NOCTOR, M. e BAINS, B. Teaching controversial issues. London: Arnold, 1984. 
TJOSVOLD, D., JOHNSON, D. e LERNER, J. Effects of affirmation of one's competence, personal acceptance, and disconfirmation of one's competence on incorporation of opposing information on problem-solving. Journal of Social Psychology, 114, p. 103-110, 1981.

WANG, H. e SCHMIDT, W. History, philosophy and sociology of science in science education: Results from the third international mathematics and science study. Science and Education, 10, p. 51-70, 2001.

WELLINGTON, J. (Ed.). Controversial issues in the curriculum. Oxford: Basil Blackwell, 1986.

YERRICK, R. Lower track science students' argumentation and open inquiry instruction. Journal of Research in Science Teaching, 37, p. 807-838, 2000.

ZEIDLER, D.L. The role of moral reasoning on socioscientific issues and discourse in science education. Dordrecht: Kluwer Academic Press, 2003.

e KEEFER, M. The role of moral reasoning and the status of socioscientific issues in science education. In: ZEIDLER, D.L. (Ed.). The role of moral reasoning on socioscientific issues and discourse in science education. Dordrecht: Kluwer Academic Press, 2003, p. 7-33.

e LEWIS, J. Unifying themes in moral reasoning on socioscientific issues and discourse. In: ZEIDLER, D.L. (Ed.). The role of moral reasoning on socioscientific issues and discourse in science education. Dordrecht: Kluwer Academic Press, 2003, p. 289-306.

; WALKER, K.; ACKETT, W. e SIMMONS, M. Tangled up views: Beliefs in the nature of science and responses to socioscientific dilemmas. Science Education, 86(3), p. 343-367, 2003.

ZOHAR, A. e NAMET, F. Fostering students' knowledge and argumentation skills through dilemmas in human genetics. Journal of Research in Science Teaching, 39, p. 35-62, 2002. 